\title{
MONO-COMPONENTS VS IMFS IN SIGNAL DECOMPOSITION
}

\author{
TAO QIAN* ${ }^{*}$ and LIMING ZHANG ${ }^{\dagger}$ \\ University of Macau, P.O. Box 3001, Macau \\ ${ }^{*}$ stttq@umac.mo \\ †lmzhang@umac.mo \\ HONG LI \\ Huazhong University of Science and Technology \\ Wuhan 430074, China \\ lhlh0125@163.com
}

Received 18 January 2007

Revised 15 November 2007

\begin{abstract}
The concepts of intrinsic mode functions and mono-components are investigated in relation to the empirical mode decomposition. Mono-components are defined to be the functions for which non-negative analytic instantaneous frequency is well defined. We show that a great variety of functions are mono-components based on which adaptive decomposition of signals are theoretically possible. We justify the role of empirical mode decomposition in signal decomposition in relation to mono-components.
\end{abstract}

Keywords: Instantaneous frequency; Hilbert transform; mono-component; empirical mode decomposition; intrinsic mode functions; Hilbert-Huang transform.

AMS Subject Classification: 30D55, 31A20, 31C05, 42A50, 42B20

\section{Introduction}

In Ref. 6 and the related literature, an iterative algorithm for decomposing signals, called empirical mode decomposition (EMD), is proposed and implemented to practical problems. The algorithm procedure results a certain type of functions, called intrinsic mode functions (IMFs) that do not have a mathematical definition but have the following properties:

(i) Between every pair of consecutive local extremal points there is exactly one zero-crossing; and

(ii) it exhibits zero "local mean".

The condition (i) is exact, but (ii) is not. Condition (ii) depends on the types of the functions (e.g. splines) adopted in the construction of "envelope functions" in 
the EMD algorithm. A function is or is not an IMF, as a matter of fact, has to be in accordance with the certain EMD algorithm adopted. The only way to show a function to be an IMF of a certain EMD is to show that it cannot be further decomposed by the EMD. The EMD algorithm adopted by the present paper is based on a filter bank developed by G. Rilling and his collaborators, with the last update in May 2005 (run by Matlab 6.1).

The initiates of EMD expected that each IMF, denoted by $\psi$, is well-behaved with Hilbert transformation, and has "instantaneous frequency". This requires that there is a phase-amplitude modulation for $\psi$, ie.

$$
\psi(t)=\rho(t) \cos \theta(t)
$$

that satisfies the Hilbert transform condition

$$
H[\rho \cos \theta](t)=\rho \sin \theta
$$

and, furthermore,

$$
\theta^{\prime}(t) \geq 0, \quad \text { a.e. }
$$

Requirement (1.2) in relation to Hilbert transformation can be easily satisfied for any signal with finite energy (i.e. $\psi \in L^{2}$ ). It involves the construction of the associated analytic signal, $A \psi$, through the Hilbert transform $H \psi$, viz.

$$
A \psi(t)=\psi(t)+i H \psi(t)=\rho(t) e^{i \theta(t)},
$$

and, therefore,

$$
\psi(t)=\rho(t) \cos \theta(t), \quad H \psi=H[\rho \cos \theta]=\rho \sin \theta .
$$

We call the amplitude-phase modulation obtained via the associated analytic signal the analytic modulation, and the corresponding amplitude and phase functions the analytic amplitude and analytic phase functions, respectively. It may be shown, via a direct computation using the property $H^{2}=-I, I$ being the identity operator, and (1.2) is equivalent to the eigenfunction condition

$$
H\left(\rho e^{i \theta}\right)=-i \rho e^{i \theta} .
$$

It is easy to show that the converse is also true: If (1.1) and (1.2) hold, then $\rho$ and $e^{i \theta}$ have to coincide with those obtained through the associated analytic signal based on Hilbert transformation.

We note that for signals with finite energy the associated analytic signal is uniquely determined, and so are the associated analytic amplitude and phase. We say that the analytic instantaneous frequency is defined if and only if the analytic phase function $\theta(t)$ enjoys the property (1.3), and in the case the analytic instantaneous frequency is defined to be $\theta^{\prime}(t)$. The requirement (1.3) is based on the theory and practice of signal analysis. These Hilbert-transformation-related concepts are closely related to the so called physically realizable signals in physics, and analytic (holomorphic) functions in complex analysis, in particular the functions in the Hardy $H^{p}$ spaces. 
Turning back to the IMFs, one finds that one cannot expect the relation (1.3) to hold for the associated analytic phase functions of the IMFs in general. In Ref. 15, certain IMFs are constructed to show that their analytic instantaneous frequency does not exist: The derivative $\theta^{\prime}(t)$ in some cases changes sign in adjacent intervals. Reference 15, on the other hand, proposes an alternative of this situation. The authors call the functions that satisfy the above condition (i) weak-IMF, and show that if a function $f$ is $C^{2}[a, b]$, and both $f$ and its derivative $f^{\prime}$ have only simple zeros, then $f$ is a weak-IMF if and only if it is a solution of a certain self-adjoint ODE

$$
\left(P f^{\prime}\right)^{\prime}+Q f=0
$$

where $P$ and $Q$ are some differentiable and positive functions. Indeed, under the Prüfer substitution $P(t) f^{\prime}(t)=\rho(t) \cos \theta(t), f(t)=\rho(t) \sin \theta(t)$ to solve the equation, we have $\theta^{\prime}(t)>0$, and hence $f$ has a monotone phase and possess positive instantaneous frequency $\theta^{\prime}(t)$. Such defined instantaneous frequency from the Prüfer substitution is, as a matter of fact, a non-analytic instantaneous frequency. That is to say, by expressing a weak-IMF $f$ as a Prüfer-solution of a self-adjoint ODE with the form

$$
f(t)=\rho(t) \cos \theta_{1}(t),
$$

we have a positive frequency $\theta_{1}^{\prime}$, However,

$$
H f(t) \neq \rho(t) \sin \theta_{1}(t),
$$

in general. That is, for a general weak IMF, (1.3) holds but (1.2) does not. On the other hand, the analytic modulation of any signal of finite energy satisfies (1.2) but may not for (1.3). This describes the situation as stated in the traditional Chinese philosophy: "One cannot get fish and bear's paw at the same time" (Mencius). The authors $^{15}$ subsequently suggest "In the final analysis, practitioners in signal processing will make the decision on when the use of analyticity is appropriate, and to what extend a non-monotone phase is necessary". Apart from lack of analyticity, another draw-back of the Prüfer- "instantaneous frequency" is: The positive instantaneous frequency is not determined by the signal itself. Indeed, in producing such an instantaneous frequency, through an ODE and its solution, one depends on a randomly chosen envelop of the original signal from a wide class of functions (see the proof of Theorem 3.1 of Ref. 15 on the selection of the envelop function $R$ ). The method of Ref. 15 in this regards, like EMD, is considered as an experimental method.

The success of EMD is its effectiveness to decompose signals with ample applications. From the localization point of view it has a great advantage. The controversy for the method arises from the fact that it cannot define mathematical concepts independently of the algorithm, and the result of the decomposition depends on the algorithm but not only on the signal. There exist variations to the commonly used EMD method. An IMF is a weak-IMF that is theoretically a solution of a 
certain self-adjoint ODE, while self-adjoint ODEs describe almost all wave-looking (weak-IMFs) signals that fail to possess good and basic analytic properties.

The purpose of the present paper is to stick on the analyticity, and to show that there does exist a large family of functions for which both the conditions (1.2) and (1.3) are satisfied. Thus, in signal processing, one does at the same time get both fish and bear's paw. The functions satisfying simultaneous (1.2) and (1.3) are called mono-components $(\mathrm{MCs})^{10}$ that are identical to the functions having analytic instantaneous frequencies (also see next section). The family contains the usual trigonometric functions, and many more. Therefore it does not form an orthogonal base in $L^{2}$. It is vastness of the family that makes adaptive decomposition of signals possible. The decomposition asserts that functions are composed by pieces with globally analytic properties as real parts of functions in the Hardy spaces, and with locally well-defined instantaneous frequency. The adaptivity addresses fast approximation.

This paper contains some experimental results to test the effectiveness of the decomposition through the EMD algorithm in relation to mono-components. Mathematically mono-components are basic building blocks that they cannot be further decomposed into more basic units that possess the same analysis and physics properties. It would be interesting, and, in fact, desired to see the effectiveness of EMD in decomposing sums into mono-components from which they are composed. Before any experiment is carried through, one can already answer the question by "No", for the resulted functions of EMD must all be its IMFs, while mono-components are not necessarily IMFs. It is not surprising that if a sum is already an IMF but not a MC, then it cannot be further decomposed. It is also not surprising that mono-components, as fundamental building blocks, can still be further decomposed by EMD. Nevertheless, it is rather promising that EMD in many cases can decompose the sums into some IMF pieces of which each may correspond to a composing mono-component, with the same locations for the same types of extreme as in the corresponding mono-component. In those cases one could say that EMD modifies the composing mono-components into its own IMFs.

The main body of the paper is divided into three parts. In Sec. 2, we, following Refs. 12, 8-10, 4 and 13, but in a self-contained manner, discuss mono-components from the theoretical point of view. Theorem 2.1 concerns simple comparisons with between the classes IMFs and MCs. We indicate the position of the class of the continuous mono-components in the Banach algebra of the continuous functions that provides some insights for adaptive decomposition by using the mono-components. In Sec. 3, we construct certain mono-components of the particular form $\rho e^{i \theta}$ where the part $e^{i \theta}$ itself is a mono-component. The results obtained in the section are new that depend on some new developments of Bedrosian's theorem. We provide explicit formulas for all types of working examples in the paper for engineering use. Figures for visualizing the constructed functions are provided. In Sec. 4 we justify the role of EMD in relation to mono-components. 


\section{Mono-Components}

The terminology "mono-component" appeared in literature ${ }^{2}$ without a precise mathematical definition. In Ref. 10, we propose the terminology for the functions with modulation (1.1) and satisfy simultaneously (1.2) and (1.3). In the terminology used in the previous section, a function is a mono-component if and only if it possesses analytic instantaneous frequency.

Denote by $\mathcal{W} \mathcal{I} \mathcal{F} \mathcal{M}$ the class of weak-IMFs, by $\mathcal{I F M}$ the class of IMFs, and $\mathcal{M C}$ the class of MCs, i.e. mono-components.

It is convenient to also adopt complex-valued signals. We proved in Ref. 9 that for $f \in L^{p}, 1 \leq p \leq \infty$, with the expression $f=\rho(c+i s)$, where $\rho \geq 0, c$ and $s$ are real-valued with $c^{2}+s^{2}=1$, then $H(\rho c)=\rho s$ if and only if $f$ is the boundary value of a function in the complex Hardy $H^{p}$ space. The relation $H(\rho c)=\rho s$ is equivalent to the eigenvalue condition $H f=-i f$. In below $H^{\infty}$ is of a particular interest. A function $f$ in the upper-half-complex plane or in the unit disc, $\mathbf{D}$, is said to be in $H^{\infty}$ if it is holomorphc and bounded in the respective regions. A function $f \in L^{p}$ is a complex-valued mono-component if and only if $H f=-$ if and there exists a modulation $f=\rho(t) e^{i \theta(t)}$ with the property $\theta^{\prime}(t) \geq 0$, a.e. Obviously there exists a one-to-one corresponding relationship between real mono-components and complex mono-components. Based on this we use the same notation $\mathcal{M C}$ for the classes of real and complex-valued mono-components. For complex-valued signals there exists a dual concept: We say that $f$ is a dual-complex mono-component, if $H f=$ if and there exists a modulation $f=\rho(t) e^{i \theta(t)}$ such that $\theta^{\prime}(t) \leq 0$, a.e. It is easy to show ${ }^{9}$ that $\rho(t) e^{i \theta(t)}$ is a dual mono-component if and only if $\rho(t) e^{-i \theta(t)}$ is a mono-component. For this reason we can only concentrate in studying monocomponents.

There is a counterpart theory for signals defined on the unit circle, $\mathbf{T}$, that corresponds to periodic signals on the real line. We call complex mono-components and dual complex mono-components on the circle circular mono-components and circular dual-mono-components, respectively. In Refs. 9 and 10, we cite two methods to extend the theory on the circle to that on the real line. One is $2 \pi$-periodic extension, and the other is via Cayley transformation. In below, except otherwise stated, functions defined on the circle are also considered as periodic functions on the line. On the unit circle we use circular Hilbert transformation in place of the standard Hilbert transformation on the line, denoted by $\tilde{H}$. It has a singular integral expression and a Fourier multiplier expression. ${ }^{8,12}$ We note that if $f \in$ $L^{2}(\mathbf{T})$ on the circle and $f(t)=\sum_{k=-\infty}^{\infty} c_{k} e^{i k t}$ is its Fourier expansion, then $\tilde{H} f=$ $-i \sum_{k=-\infty}^{\infty} \operatorname{sgn}(k) c_{k} e^{i k t}$. As consequence, for $f \in H^{2}(\mathbf{T})$, with the expansion $f(t)=$ $\sum_{k=0}^{\infty} c_{k} e^{i k t}$, there follows $\tilde{H} f=-i f+i c_{0}$, where $c_{0}$ is the average of the function on the circle: $c_{0}=\frac{1}{2 \pi} \int_{0}^{2 \pi} f\left(e^{i t}\right) d t$.

It follows that with the analytic modulation $f=\rho e^{i \theta}$ we have

$$
\tilde{H}(\rho \cos \theta)=\rho \sin \theta-\operatorname{Im}\left(c_{0}\right), \quad \tilde{H}(\rho \sin \theta)=-\rho \cos \theta+\operatorname{Re}\left(c_{0}\right) .
$$


Due to this relation, a complex-valued function $f \in H^{\infty}(\mathbf{T})$ with zero moment $c_{0}=0$ is a circular mono-component if and only if $H(f)=-i f$, and the modulation $f=\rho e^{i \theta}$ has a determined argument parametrization $\theta=\theta(t)$ such that $\theta^{\prime} \geq 0$.

In the sequel two conventions will be adopted. One is to abbreviate $\tilde{H}$ as $H$. Thus, $H$ being the standard or circular Hilbert transformation will depend on the context. There is no ambiguity in doing so, as for signals defined on the finite interval $[0,2 \pi)$ (viz. the unit circle) or those $2 \pi$-periodically defined on the real line, their Hilbert transforms coincide when restricted to the interval $[0,2 \pi) .{ }^{10}$ The second convention is, when $f$ is in the Hardy $H^{2}$ of the unit disc with $c_{0}=0$, we have $H f=-i f$, and we regard this case as the exact identity or the exact eigenfunction relation in the text.

There is a fundamental subclass of $\mathcal{M C}$ called Fourier atoms. Fourier atoms are first studied in the twin Refs. 8 and 12, and subsequently in Ref. 3 , and then in Refs. 9, 4 and others. The real-line version of Fourier atoms and their products were studied in by Picinbono. ${ }^{7}$ On the circle they are the boundary values of the Möbius transforms

$$
\tau_{a}(z)=\frac{z-a}{1-\bar{a} z}, \quad|a|<1
$$

A Fourier atom has the form

$$
e^{i \theta_{a}(t)}=\frac{e^{i t}-a}{1-\bar{a} e^{i t}}, \quad t \in[0,2 \pi),
$$

where

$$
\frac{\theta_{a}^{\prime}(t)}{2 \pi}=\frac{1}{2 \pi} \frac{1-|a|^{2}}{1-2|a| \cos t+|a|^{2}}>0
$$

is the Poisson kernel at $a$ of the unit circle. We have the explicit formulas

$$
\begin{aligned}
& \cos \theta_{a}(t)=\frac{\cos t-2|a| \cos t_{a}+|a|^{2} \cos \left(t-2 t_{a}\right)}{1-2|a| \cos \left(t-t_{a}\right)+|a|^{2}}, \\
& \sin \theta_{a}(t)=\frac{\sin t-2|a| \sin t_{a}+|a|^{2} \sin \left(t-2 t_{a}\right)}{1-2|a| \cos \left(t-t_{a}\right)+|a|^{2}} .
\end{aligned}
$$

Basic mono-components are univalent or multivalent functions among which Möbius transforms are considered to be the very fundamental building blocks. They have deep involvement in both applied and theoretical mathematics and physics. The class of Fourier atoms is denoted by $\mathcal{F} \mathcal{A}$. The class of the real parts of the functions in $\mathcal{F} \mathcal{A}$, that are functions $\cos \theta_{a}(t)$, is still denoted by $\mathcal{F} \mathcal{A}$. There will be no confusion from the context. We note that, for a Fourier atom, $e^{i \theta_{a}(t)}$, there hold ${ }^{9}$

$$
H\left(\cos \theta_{a}\right)=\sin \theta_{a}+\operatorname{Im}(a), \quad H\left(\sin \theta_{a}\right)=-\cos \theta_{a}-\operatorname{Re}(a) .
$$

If, in particular, $a$ is real, being denoted by $a=r \in(-1,1)$ (see Sec. 3), then there hold

$$
H\left(\cos \theta_{r}\right)=\sin \theta_{r}, \quad H\left(\sin \theta_{r}\right)=-\cos \theta_{r}-r
$$

where the first relation is exact. 

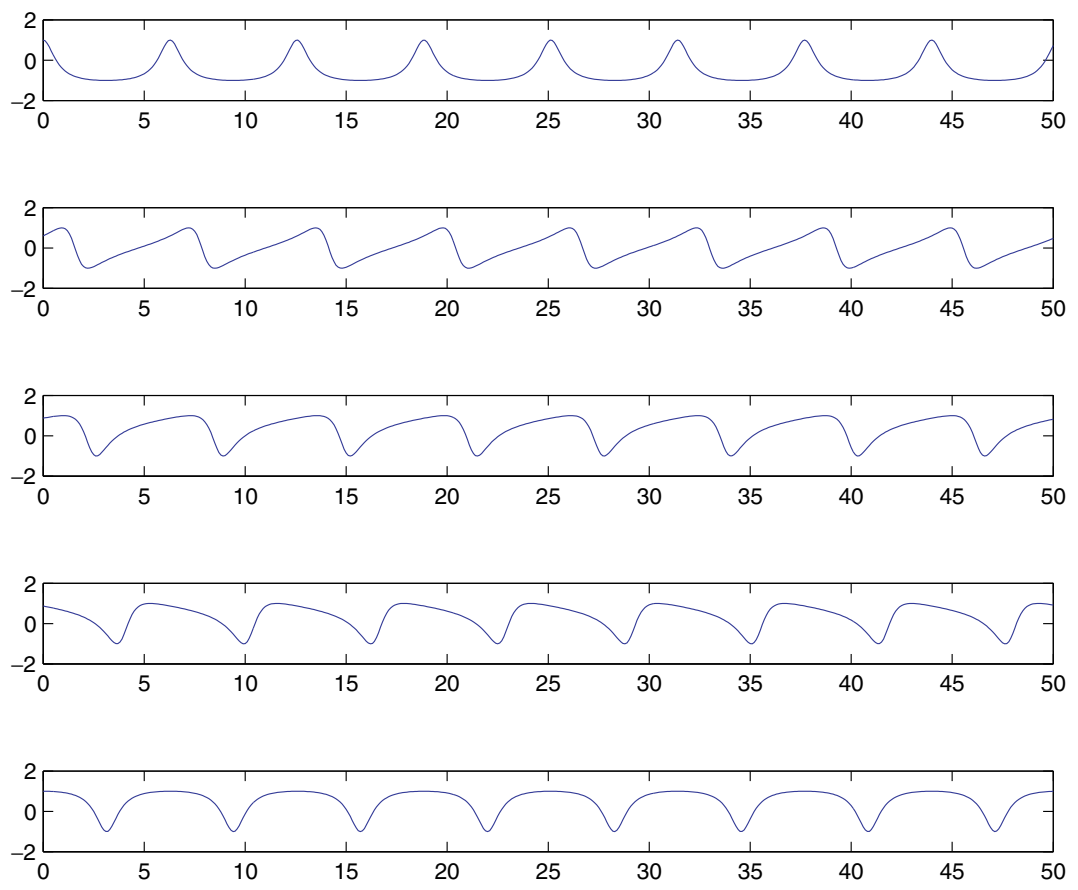

Fig. 1. Graphs of Fourier atoms.

Figure 1 presents graphs of the Fourier atoms $\cos \theta_{a}(t)$ for $a=1 / 2, i / 2$, $(1 / 2) e^{i \frac{3 \pi}{4}},(1 / 2) e^{-i \frac{3 \pi}{4}}$ and $-1 / 2$.

Finite products of Fourier atoms, or equivalently, finite Blaschke products, are mono-components. This follows from the fact that a finite product of Möbius transforms is a bounded analytic function in the disc, and the phase of its boundary value is the finite sum of the phases of the corresponding Fourier atoms. In particular, trigonometric functions $e^{\text {int }}$ are mono-components. This "finite product principle" can be carried out to any finite products of mono-components in the $H^{p}$ spaces.

A larger class than that of Fourier atoms, still being fundamental, consists of boundary values of starlike functions. ${ }^{10}$ We call a function $f(z)$ a starlike function if it is a univalent conformal mapping from the unit disc to a starlike domain with pole 0 and $f(0)=0$. The requirement $f(0)=0$ being the pole is not essential. If $|a|<1$ and $f(a)=b$ is the pole of the image starlike domain, then $g(z)=$ $f\left(\tau_{-a}(z)\right)-b$ maps univalently and conformally the unit disc $\mathbf{D}$ to a starlike domain with $g(0)=0$ being the pole. One has $f(z)=g\left(\tau_{a}(z)\right)+b$, and, if $g$ is a monocomponent with the modulation $g\left(e^{i t}\right)=\rho(t) e^{i \theta(t)}$, then $\rho\left(\theta_{a}(t)\right) e^{i \theta\left(\theta_{a}(t)\right)}$ is a monocomponent, and $f\left(e^{i t}\right)=\rho\left(\theta_{a}(t)\right) e^{i \theta\left(\theta_{a}(t)\right)}+b$. For practical reasons we restrict ourselves to the easily computed cases where the boundaries of the starlike domains are bounded rectifiable closed Jordan curves (in Ref. 10 it is phrased as "the Jordan boundary curves", see below). It may be easily proved that such starlike functions 
are bounded holomorphic functions whose boundary values are of the form $\rho(t) e^{i \theta(t)}$, with $\theta^{\prime}(t) \geq 0$, and therefore are mono-components. In Ref. 10, it is shown that the boundary values of such starlike functions are equivalent to mono-components with the zero moment and the total variation of the phase functions are $2 \pi$. The cases where $\rho$ is identical to 1 are said to be unimodular, and, otherwise, non-unimodular. It is shown in Ref. 8 that the unimodular mono-components of $2 \pi$-total variation are Fourier atoms. Similar to finite Blaschke products, finite products of boundary values of starlike functions are mono-components.

We now give an example of non-unimodular mono-components. As derived in Ref. 10 , for $a \in \mathbf{D}$, the mappings

$$
f_{a}\left(r e^{i t}\right)=\frac{r e^{i t}}{1-\bar{a} r e^{i t}}, \quad r \in\left(\frac{-1}{|a|}, \frac{1}{|a|}\right),
$$

provide the Circle Family as boundary values of certain starlike functions. For $a=|a| e^{i t_{a}}$, it is easy to deduce, with the modulation $f_{a}\left(r e^{i t}\right)=\rho(t) e^{i \theta(t)}$,

$$
\begin{aligned}
\rho(t) \cos \theta(t) & =\frac{r \cos t-|a| r^{2} \cos t_{a}}{1-2|a| r \cos \left(t-t_{a}\right)+|a|^{2} r^{2}}, \\
\rho(t) \sin \theta(t) & =\frac{r \sin t-|a| r^{2} \sin t_{a}}{1-2|a| r \cos \left(t-t_{a}\right)+|a|^{2} r^{2}} .
\end{aligned}
$$

In Fig. 2 we provide the graphs of the function $\rho(t) \cos \theta(t)$ for $a=1 / 4, r=1 / 2$ (Fig. 2(a)); $a=i / 4, r=1$ (Fig. 2(b)); and $a=1 / 4 e^{i 3 \pi / 4}, r=2$ (Fig. 2(c)). In Fig. 2(d), we exhibit the corresponding boundary curve of the starlike function of the last example. Note that the origin does not lie in the center of the image disc showing that the mono-components in the circle family are non-unimodular.

There are simple relations between the classes $\mathcal{I} \mathcal{M F}, \mathcal{W} \mathcal{I} \mathcal{M F}, \mathcal{M C}$ and $\mathcal{F} \mathcal{A}$, shown in

Theorem 2.1. We have

(i) $\mathcal{I} \mathcal{M F} \subset \mathcal{W} \mathcal{I} \mathcal{M F}$;

(ii) $\mathcal{F} \mathcal{A} \subset \mathcal{I} \mathcal{M F}$;

(iii) $\mathcal{I} \mathcal{M F} \backslash \mathcal{M C} \neq \emptyset$;

(iv) $\mathcal{M C} \backslash \mathcal{I} \mathcal{F} \mathcal{M} \neq \emptyset$; and

(v) $\mathcal{F} \mathcal{A} \subset \mathcal{I} \mathcal{M F} \cap \mathcal{M C}$.

Proof. (i) Is obvious from the definitions of the two classes. (ii) is based on the strict monotone property of $\theta_{a}$ and the basic properties of the trigonometric cos. Indeed all Fourier atoms cannot be further decomposed by EMD (see Fig. 8) and therefore are IMFs. (iii) is referred to the examples constructed in Ref. 15. To prove (iv), we need to show that there exist mono-components that can be further decomposed by EMD into more than one IMF pieces. We show this by the two examples in Fig. 10. (v) is a consequence of (ii) and the fact that $\mathcal{F} \mathcal{A}$ is contained in $\mathcal{M C}$. The proof is complete. 


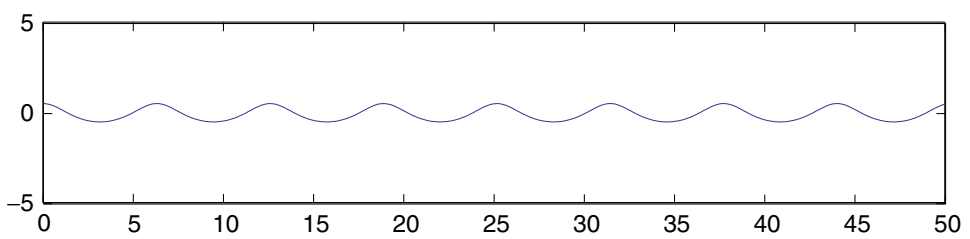

(a)

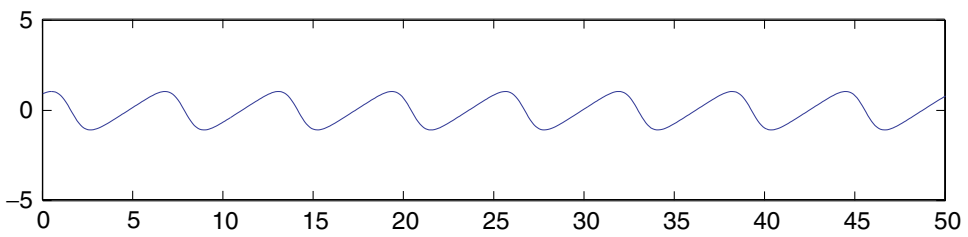

(b)

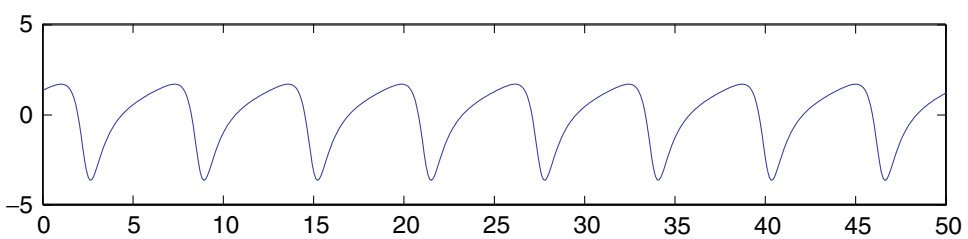

(c)

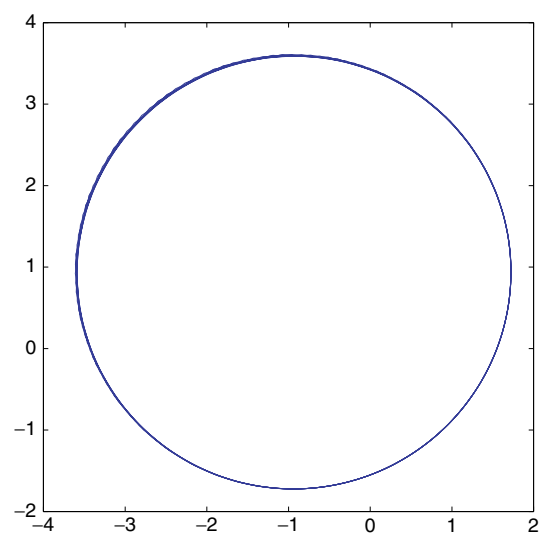

(d)

Fig. 2. The Circle family and the corresponding starlike boundary.

Denote by $\mathbf{D}$ the unit disc and $\mathbf{T}$ the unit circle in the complex plane. Let $C(\mathbf{T})$ be the Banach space of continuous functions on $\mathbf{T}$ equipped with the norm

$$
\|f\|=\sup \{|f(z)|: z \in \mathbf{T}\} .
$$

$C(\mathbf{T})$ is a commutative Banach algebra over the field of complex numbers. In particular, it satisfies $\|f g\| \leq\|f\|\|g\|$. Denote by $A(\mathbf{T})$ the sub-algebra of $C(\mathbf{T})$ consisting 
of the functions that can be continuously extended to a holomorphic function in D. $A(\mathbf{T})$ is also a Banach algebra, and, in fact, a closed sub-algebra of $C(\mathbf{T})$. For functions in $C(\mathbf{T})$ the following three conditions are equivalent:

(i) $f \in A(\mathbf{T})$;

(ii) $f$ can be uniformly approximated on $\partial \mathbf{T}$ by polynomials in $z$; and

(iii) the Fourier coefficients $c_{n}(f)$ of negative index $n$ all vanish.

Denote by $\mathcal{M C}_{C}$ the class of the functions on $\mathbf{T}$ in $\mathcal{M C} \cap C(\mathbf{T})$ adopting the norm of $C(\mathbf{T})$. We have

Theorem 2.2. (i) $\mathcal{M C}_{C}$ is closed under multiplication;

(ii) $\mathcal{M C}_{C}$ is a closed subset of $A(\mathbf{T})$;

(iii) $\mathcal{M C}_{C}$ is not closed under addition, and thus is not a sub-algebra of $A(\mathbf{T})$;

(iv) The span of $\mathcal{M C}_{C}$ is dense in $A(\mathbf{T})$; and

(v) Boundary values of starlike functions with the Jordan boundary curves belong to $\mathcal{M C}_{C}$.

Proof. (i) Assume that $f, g \in \mathcal{M C}_{C}$. Since $f, g \in L^{\infty}$ and satisfy $H f=-i f, H g=$ $-i g$, we have $f, g \in H^{\infty}$. ${ }^{9}$ Therefore, $f g \in H^{\infty}$, and hence $H(f g)=-i f g$. Now since the analytic phase functions of $f$ and $g$ both are non-decreasing, that of $f g$ as the sum of those phase functions is also non-decreasing. Therefore, $f g \in \mathcal{M C}_{C}$.

(ii) If $f \in \mathcal{M C}_{C}$, then $f \in H^{\infty} \subset H^{2}$. ${ }^{9}$ Its Fourier coefficients of negative indices are all zero, and hence $f \in A(\mathbf{T})$. This shows that $\mathcal{M C}_{C}$ is a subclass of $A(\mathbf{T})$. Now we show that the subclass $\mathcal{M C}_{C}$ is closed in the topology of $A(\mathbf{T})$. Since $A(\mathbf{T})$ is a closed subclass of $C(\mathbf{T})$, any Cauchy sequence $f_{n} \in \mathcal{M C}_{C} \subset A(\mathbf{T})$ has a limit $f \in A(\mathbf{T})$. We shall further show that $f \in \mathcal{M C}_{C}$. Now write $f_{n}(t)=\rho_{n}(t) e^{i \theta_{n}(t)}$ and $f(t)=\rho(t) e^{i \theta(t)}$. Due to $f_{n} \rightarrow f$, we have, uniformly, $\rho_{n}(t) \rightarrow \rho(t)$ and $e^{i \theta_{n}(t)} \rightarrow e^{i \theta(t)}$. Using the Ascoli-Aezelá Theorem to the convergent sequence $e^{i \theta_{n}(t)}$, we obtain the equi-continuity for $e^{i \theta_{n}(t)}$ and thus that for the sequence $\theta_{n}(t)$ as well. This enables us to choose a common branch of argument for all $\theta_{n}(t)$ and $\theta(t)$ so that $\theta_{n}(t)$ uniformly converges to $\theta(t)$. The non-decreasing of $\theta_{n}(t)$ then implies that of $\theta(t)$. Therefore $f \in \mathcal{M C}_{C}$.

(iii) We show that $\mathcal{M C}_{C}$ is not closed under the addition operation. This is a well-known fact in the study of existence of analytic instantaneous frequency. For the self-contained purpose we still construct an example. Let $f_{1}(z)=$ $n_{1} z^{m_{1}}, f_{2}=n_{2} z^{m_{2}}$, where $n_{1}, n_{2}, m_{1}, m_{2}$ are positive integers. Clearly, $f_{1}$ and $f_{2}$ both are in $\mathcal{M C}_{C}$. Set

$f\left(e^{i t}\right)=f_{1}\left(e^{i t}\right)+f_{2}\left(e^{i t}\right)=\left(n_{1} \cos m_{1} t+n_{2} \cos m_{2} t\right)+i\left(n_{1} \sin m_{1} t+n_{2} \sin m_{2} t\right)$.

Then the analytic phase function $\theta(t)$ of $f$ will satisfy

$$
\tan \theta(t)=\frac{n_{1} \sin m_{1} t+n_{2} \sin m_{2} t}{n_{1} \cos m_{1} t+n_{2} \cos m_{2} t} .
$$


Taking derivative with respect to $t$ to both sides of the above equality,

$$
\frac{\theta^{\prime}(t)}{\cos ^{2} \theta(t)}=\frac{m_{1} n_{1}^{2}+m_{2} n_{2}^{2}+\left(m_{1}+m_{2}\right) n_{1} n_{2} \cos \left(m_{1} t-m_{2} t\right)}{\left(n_{1} \cos m_{1} t+n_{2} \cos m_{2} t\right)^{2}} .
$$

If, in particular, we choose $m_{1}=3, n_{1}=1, m_{2}=1, n_{2}=2$, then the above relation becomes

$$
\frac{\theta^{\prime}(t)}{\cos ^{2} \theta(t)}=\frac{5+8 \cos \left(m_{1} t-m_{2} t\right)}{(\cos 3 t+2 \cos t)^{2}} .
$$

Thus $\theta^{\prime}(t)$ in some open intervals is positive and some open intervals negative. Hence $f$ does not belong to $\mathcal{M C}_{C}$.

(iv) The density of finite spans of $\mathcal{M C}_{C}$ is obvious as it contains all polynomials while the latter is dense in $A(\mathbf{T})$.

(v) Such a starlike function maps $\mathbf{T}$ into a bounded rectifiable close Jordan curve, and thus is absolutely continuous. The boundary value of a starlike function is also a mono-component. Therefore $f$ is in $\mathcal{M C}_{C}$. The proof of the theorem is complete.

There is no regret that $\mathcal{M C}_{C}$ is not an algebra and thus does not coincide with $A(\mathbf{T})$. By the theorem, functions in $A(\mathbf{T})$, and thus those in $H^{p}, 1 \leq p<\infty$, are all limits of finite spans of the mono-components in $\mathcal{M C}_{C}$ in the respective function spaces. The class $\mathcal{M C}_{C}$, that contains the trigonometric system, is not an orthogonal basis. It is the vastness of the class mono- and anti-mono-components that makes the adaptive decomposition of signals possible.

The adaptive decomposition is of the form

$$
f(t)=\sum_{k=1}^{N} \rho_{k}(t) e^{i \theta_{k}(t)}+r_{N}(t)
$$

where $\rho_{k}(t) \geq 0$ and $H\left(\rho_{k}(\cdot) e^{i \theta_{k}(\cdot)}\right)=\mp i \rho_{k}(\cdot) e^{i \theta_{k}(\cdot)}, \rho_{k} \geq 0$, and $\pm \theta_{k}^{\prime}(t) \geq 0$, respectively, or equivalently, with the real form,

$$
f(t)=\sum_{k=1}^{N} \rho_{k}(t) \cos \theta_{k}(t)+r_{N}(t)
$$

where $H\left(\rho_{k}(\cdot) \cos \theta_{k}(\cdot)\right)=\rho_{k}(\cdot) \sin \theta_{k}(\cdot), \rho_{k} \geq 0, \theta_{k}^{\prime} \geq 0$, and $r_{N} \rightarrow 0$ rapidly in a certain measurement. Note that any multiple complex constant $C_{k}$ is with the form $C_{k}=\left|C_{k}\right| e^{i \phi_{k}}$ where $\left|C_{k}\right|$ and $\phi_{k}$ may be incorporated into $\rho_{k}$ and $\theta_{k}(t)$, respectively. Hence there is no constants shown in the decomposition formulas (2.1) and (2.2). The above two formulas both are valid in the circular context and in the real-line context. In the complex series expansion (2.1) both the classes monocomponent and anti-mono-component are needed, but in the real series expansion (2.2) anti-mono-components, corresponding to the entries of the form $\rho \sin \theta$, may 
be avoid. Indeed, there hold

$$
\rho(t) \sin \theta(t)=\cos (\theta(t)-\pi / 2)
$$

and

$$
H(\rho(\cdot) \cos (\cdot-\pi / 2))=H(\rho(\cdot) \sin \theta(\cdot))=-\rho(\cdot) \cos \theta(\cdot)=\rho(\cdot) \sin \theta(\cdot-\pi / 2),
$$

where $\frac{d}{d t}[\theta(t-\pi / 2)]=\theta^{\prime}(t-\pi / 2) \geq 0$.

\section{Non-Unimodular Mono-Components in Relation to Fourier Atoms}

The results and examples of mono-components studied in this section, falling into the scope of starlike functions of the Jordan boundary curves and their products, are of particular interests. Suggested by Bedrosian's theorem ${ }^{1,16,14}$ one looks for mono-components of the form $\rho(t) e^{i \theta(t)}$, where $\rho \geq 0$ and $e^{i \theta(t)}$ itself is a monocomponent. The basic case is that the phase function $\theta$ is the sum of a finite number of phase functions $\theta_{a_{i}}$ where $\theta_{a_{i}}$ is from the Fourier atom $e^{i \theta_{a_{i}}}, a_{i} \in \mathbf{D} .^{13,17}$

Theorem 3.1. Assume that $e^{i \theta(t)}$ is a mono-component. Then,

(i) for any real numbers $M, N, N \geq|M|$, the function

$$
\rho(t) \cos \theta(t)=(M \cos \theta(t)+N) \cos \theta(t)
$$

is a mono-component, where $N \geq|M|$; and

(ii) if $\theta(t)=\theta_{a}(t)$ is from the Fourier atom $e^{i \theta_{a}(t)}, a \in \mathbf{D}$, then all monocomponents of the form $\rho \cos \theta$ is of the form

$$
\rho(t) \cos \theta(t)=(M \cos \theta(t)+N) \cos \theta(t),
$$

where $M, N$ are real numbers with $N \geq|M|$.

Proof. (i) Since $e^{i \theta(t)}$ is a mono-component, it is the boundary value of a bounded holomorphic function in the unit disc. ${ }^{9}$ As a consequence, $e^{i 2 \theta(t)}$ is the boundary value of a bounded holomorphic function in the unit disc, and thus is also a mono-component. In particular,

$$
H[\cos (2 \theta)]=\sin (2 \theta) .
$$

We therefore have

$$
\begin{aligned}
H[(M \cos \theta(t)+N) \cos \theta(t)] & =H\left[M \cos ^{2} \theta(t)+N \cos \theta(t)\right] \\
& =H[(M / 2)(1+\cos (2 \theta(t)))+N \cos \theta(t)] \\
& =(M / 2) \sin (2 \theta(t))+N \sin \theta(t) \\
& =(M \cos \theta(t)+N) \sin \theta(t),
\end{aligned}
$$

as desired. 
(ii) Now assume that $\theta$ is from the Fourier atom $e^{i \theta_{a}}$ for some $a \in \mathbf{D}$. We need to show that any non-negative $\rho$ satisfying $H\left(\rho e^{i \theta_{a}}\right)=-i \rho e^{i \theta_{a}}$ is of the form $\rho=M \cos \theta_{a}+N, M, N$ being real numbers. If $\rho$ is of such a form and nonnegative, then we must have $N \geq|M|$. We will borrow the following general result from Ref. 13 .

Proposition 3.1. Let $\theta(t)=\theta_{r_{1}}(t)+\cdots+\theta_{r_{n}}(t), 0 \leq r_{j}<1, j=1, \ldots, n$, and $\rho(t) \geq 0$. Then $\rho(t) e^{i \theta(t)}$ is a mono-component if and only if

$$
\rho(t)=\operatorname{Re} \frac{\sum_{j=1}^{n} A_{j} e^{i j t}}{\prod_{j=1}^{n}\left(1-r_{j} e^{i t}\right)}+B,
$$

where $A_{j}, j=1, \ldots, n-1$, are complex numbers and $A_{n}$ and $B$ are real numbers chosen so that $\rho(t) \geq 0$ for all $t$.

Using the result for $n=1$ and $a=r \in[0,1)$ being real, to prove the assertion is reduced to showing that for suitable real numbers $A, B$ we have

$$
\cos \theta_{r}(t)=\operatorname{Re} \frac{A e^{i t}}{1-r e^{i t}}+B,
$$

or equivalently,

$$
\operatorname{Re} \frac{e^{i t}}{1-r e^{i t}}=M \cos \theta_{r}(t)+N
$$

for certain real numbers $M, N$. Now we show (3.3).

On one hand,

$$
\cos \theta_{r}(t)=\operatorname{Re} \frac{e^{i t}-r}{1-r e^{i t}}=\frac{\left(1+r^{2}\right) \cos t-2 r}{1-2 r \cos t+r^{2}} .
$$

On the other hand,

$$
\begin{aligned}
\operatorname{Re} \frac{A e^{i t}}{1-r e^{i t}}+B & =\frac{A \cos t-A r+B\left(1-2 r \cos t+r^{2}\right)}{1-2 r \cos t+r^{2}} \\
& =\frac{(A-2 r B) \cos t+\left[\left(1+r^{2}\right) B-A r\right]}{1-2 r \cos t+r^{2}}
\end{aligned}
$$

It therefore reduces to solving the linear system

$$
A-2 r B=1+r^{2}, \quad(-r) A+\left(1+r^{2}\right) B=-2 r
$$

for $A$ and $B$ which surely has a solution as $r \neq \pm 1$.

Now consider the case $a \neq 0$, being not positive. That is $a=r e^{i t_{a}}$, where $0<r<1, e^{i t_{a}} \neq 1$. By expressing

$$
e^{i \theta_{a}(t)}=e^{i t_{a}} e^{i \theta_{r}\left(t-t_{a}\right)},
$$

we know from the assertion (i) that all $\rho$ 's satisfying the mono-component eigenfunction requirement are of the form $\rho(t)=M \cos \theta_{r}\left(t-t_{a}\right)+N$. In particular, from 
the just proved assertion (i), there exist real numbers $M_{0} \neq 0$ and $N_{0}$ such that $\cos \theta_{a}(t)+1=M_{0} \cos \theta_{r}\left(t-t_{a}\right)+N_{0}$. Thus $\cos \theta_{r}\left(t-t_{a}\right)=M_{1} \cos \theta_{a}(t)+N_{1}$ for some real numbers $M_{1}, N_{1}$. Therefore, any form $M \cos \theta_{r}\left(t-t_{a}\right)+N$ is also of the form $M \cos \theta_{a}(t)+N$. The proof for (ii) is complete, and thus that of the theorem as well.

The theorem shows that the particular example obtained in Ref. 11 through dealing with certain meromorphic functions turns to be the general solution to the eigenfunction problem for $\theta=\theta_{a}$.

In the proof of Theorem 3.1 we also obtained an explicit formula for $\rho$ :

$$
\rho(t)=\frac{(A-2 r B) \cos t+\left[\left(1+r^{2}\right) B-A r\right]}{1-2 r \cos t+r^{2}},
$$

where $A$ and $B$ are real numbers chosen so that $\rho(t) \geq 0$.

When $a$ is not a positive real number, namely $a=r e^{i t_{a}}, 0 \leq r<1, e^{i t_{a}} \neq 1$, the formula reduces to

$$
\rho(t)=\frac{(A-2|a| B) \cos \left(t-t_{a}\right)+\left[\left(1+|a|^{2}\right) B-A|a|\right]}{1-2|a| \cos \left(t-t_{a}\right)+|a|^{2}},
$$

where $A$ and $B$ are real numbers chosen so that $\rho(t) \geq 0$.

In Fig. 3, we present graphs of mono-components corresponding to some particular values of $a, M$ and $N$ in the formula of Theorem 3.1. Figures 3(c) and 3(d) are the graphs of $\left(\left(M \cos \theta_{a}+N\right) \cos \theta_{a},\left(M \cos \theta_{a}+N\right) \sin \theta_{a}\right)$ for $a=1 / 2, M=7 / 4, N=2$ and $a=1 / 2+2 i / 3, M=-1 / 2, N=1$, respectively.

There are two remarks for Theorem 3.1.

Remark 1. Since finite products of complex mono-components are still complex mono-components, besides the formula (3.2), one also has a tensor form formula

$$
\rho(t)=\prod_{k=1} \rho_{k}(t)=\prod_{k=1}^{n}\left(M_{k} \cos \theta_{a_{k}}(t)+N_{k}\right)
$$

for $\theta=\sum_{k=1}^{n} \theta_{a_{k}}$.

Figures 4 and 5 provides examples of products of boundaries values of starlike functions. In Fig. 4, the mono-components are constructed based on Theorem 3.1 and Remark 1 for the parameters $M_{1}=1 / 2, N_{1}=2, a_{1}=1 / 2$ and $M_{2}=7 / 4$, $N_{2}=2, a_{2}=1 / 3$; and in Fig. 5 for $M_{1}=1 / 2, N_{1}=2, a_{1}=i / 2$ and $M_{2}=1 / 2$, $N_{2}=2, a_{2}=1 / 2$, and $M_{3}=-7 / 4, N_{3}=2, a_{3}=3 i / 4$.

Remark 2. Detailed analysis, ${ }^{17}$ shows that for $e^{i \phi(t)}=e^{i t} e^{i \theta_{r}(t)}, r \in(0,1), \rho \in$ $L^{2}(\mathbf{T})$, the function $\rho e^{i \phi}$ is a mono-component with the cancellation property

$$
\int_{0}^{2 \pi} \rho(t) e^{i \phi(t)} d t=0
$$

if and only if

$$
\rho(t)=\operatorname{Re} \frac{A e^{i t}}{1-r e^{i t}}+B
$$




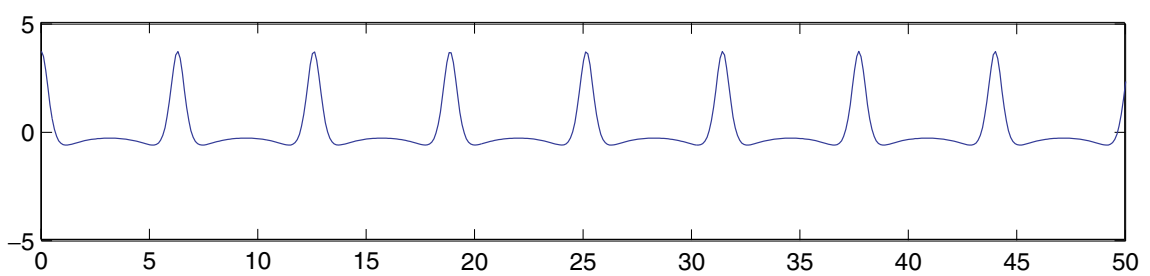

(a) $a=1 / 2, M=7 / 4, N=2$.

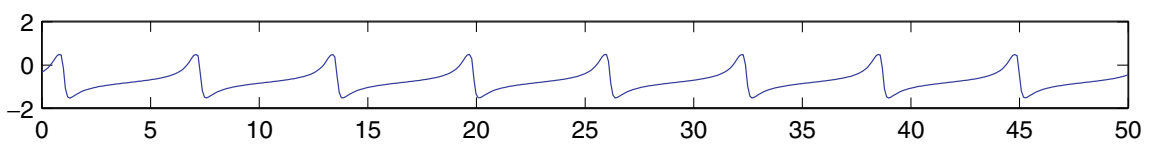

(b) $a=1 / 2+2 \mathrm{i} / 3, M=-1 / 2, N=1$.

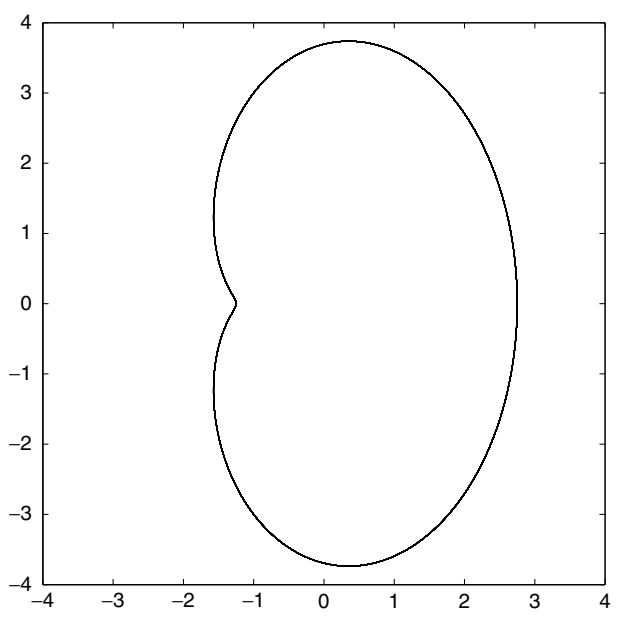

(c)

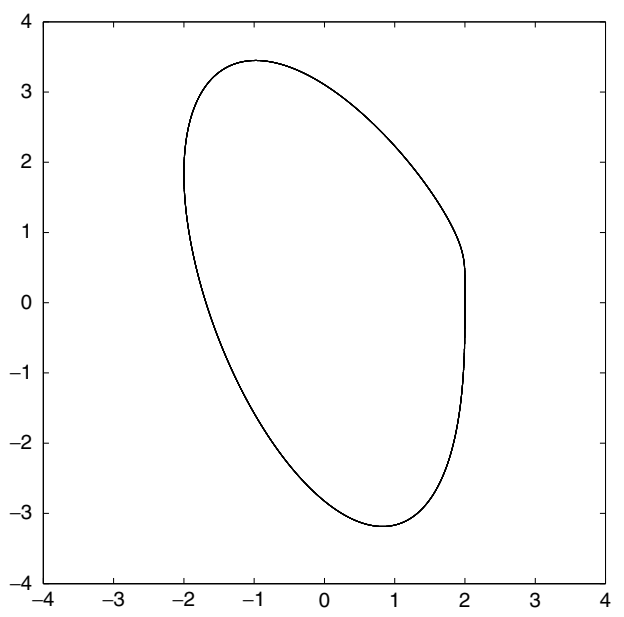

(d)

Fig. 3. Examples for mono-components of the form $\left(M \cos \theta_{a}+N\right) \cos \theta_{a}$.

where $A$ is a complex number and $B$ is a real number such that $\rho(t) \geq 0$ for all $t$.

This cannot be treated as a special case for $n=2$ in Proposition 3.1 as there was no requirement (3.5). The above formula gives

$$
\rho(t) \cos \phi(t)=\left(\rho_{0} \frac{\cos \left(\theta_{0}-t\right)-r \cos \theta_{0}}{1-2 r \cos t+r^{2}}+B\right) \frac{\cos 2 t-2 r \cos t+r^{2}}{1-2 r \cos t+r^{2}},
$$

and

$$
\rho(t) \sin \phi(t)=\left(\rho_{0} \frac{\cos \left(\theta_{0}-t\right)-r \cos \theta_{0}}{1-2 r \cos t+r^{2}}+B\right) \frac{\sin 2 t-2 r \sin t}{1-2 r \cos t+r^{2}},
$$

where the terms inside the brackets correspond to the $\rho$ part, and the constants $\rho_{0}, \theta_{0}$ and $B$ should be chosen so that $\rho \geq 0$. 

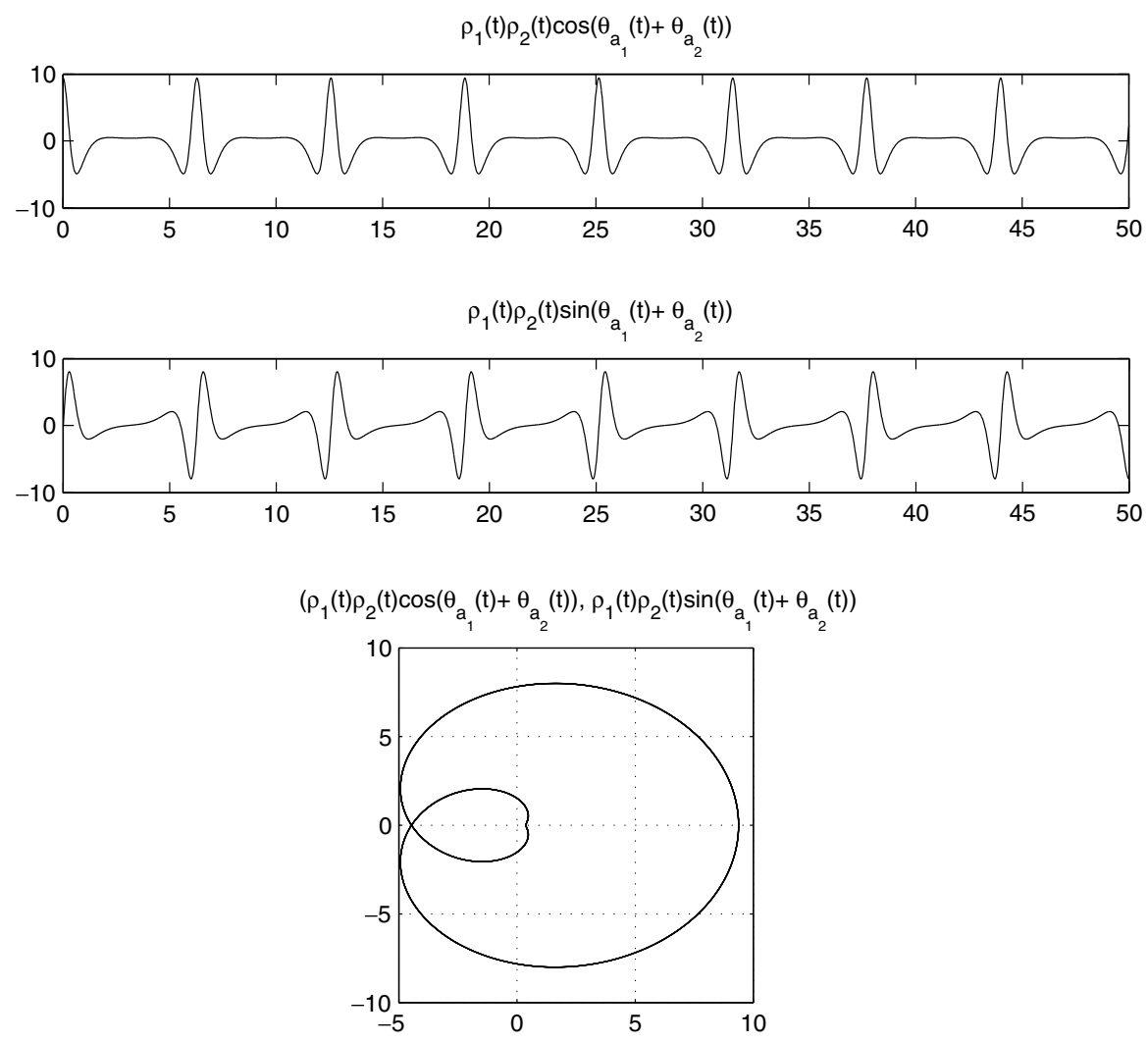

Fig. 4. Mono-component from product of two starlike functions.

For the general case $a=r e^{i t_{a}}, 0<r<1$ we use the expansion

$$
e^{i \theta_{1}(t)}=e^{i t} \tau_{a}\left(e^{i t}\right)=e^{2 i t_{a}} e^{i\left(t-t_{a}\right)} \frac{e^{i\left(t-t_{a}\right)}-r}{1-r e^{i\left(t-t_{a}\right)}},
$$

The same reasoning as before implies that $\rho_{1}(t)=\rho\left(t-t_{a}\right)$ and the counterpart formulas of (3.6) and (3.7) read

$$
\begin{aligned}
\rho_{1}(t) \cos \theta_{1}(t)= & {\left[\rho_{0} \frac{\cos \left(\theta_{0}-t+t_{a}\right)-r \cos \theta_{0}}{1-2 r \cos \left(t-t_{a}\right)+r^{2}}+B\right] } \\
& \times \frac{\cos (2 t)-2 r \cos \left(t+t_{a}\right)+r^{2} \cos \left(2 t_{a}\right)}{1-2 r \cos \left(t-t_{a}\right)+r^{2}},
\end{aligned}
$$

and

$$
\begin{aligned}
\rho_{1}(t) \sin \theta_{1}(t)= & {\left[\rho_{0} \frac{\cos \left(\theta_{0}-t+t_{a}\right)-r \cos \theta_{0}}{1-2 r \cos \left(t-t_{a}\right)+r^{2}}+B\right] } \\
& \times \frac{\sin (2 t)-2 r \sin \left(t+t_{a}\right)+r^{2} \sin \left(2 t_{a}\right)}{1-2 r \cos \left(t-t_{a}\right)+r^{2}},
\end{aligned}
$$



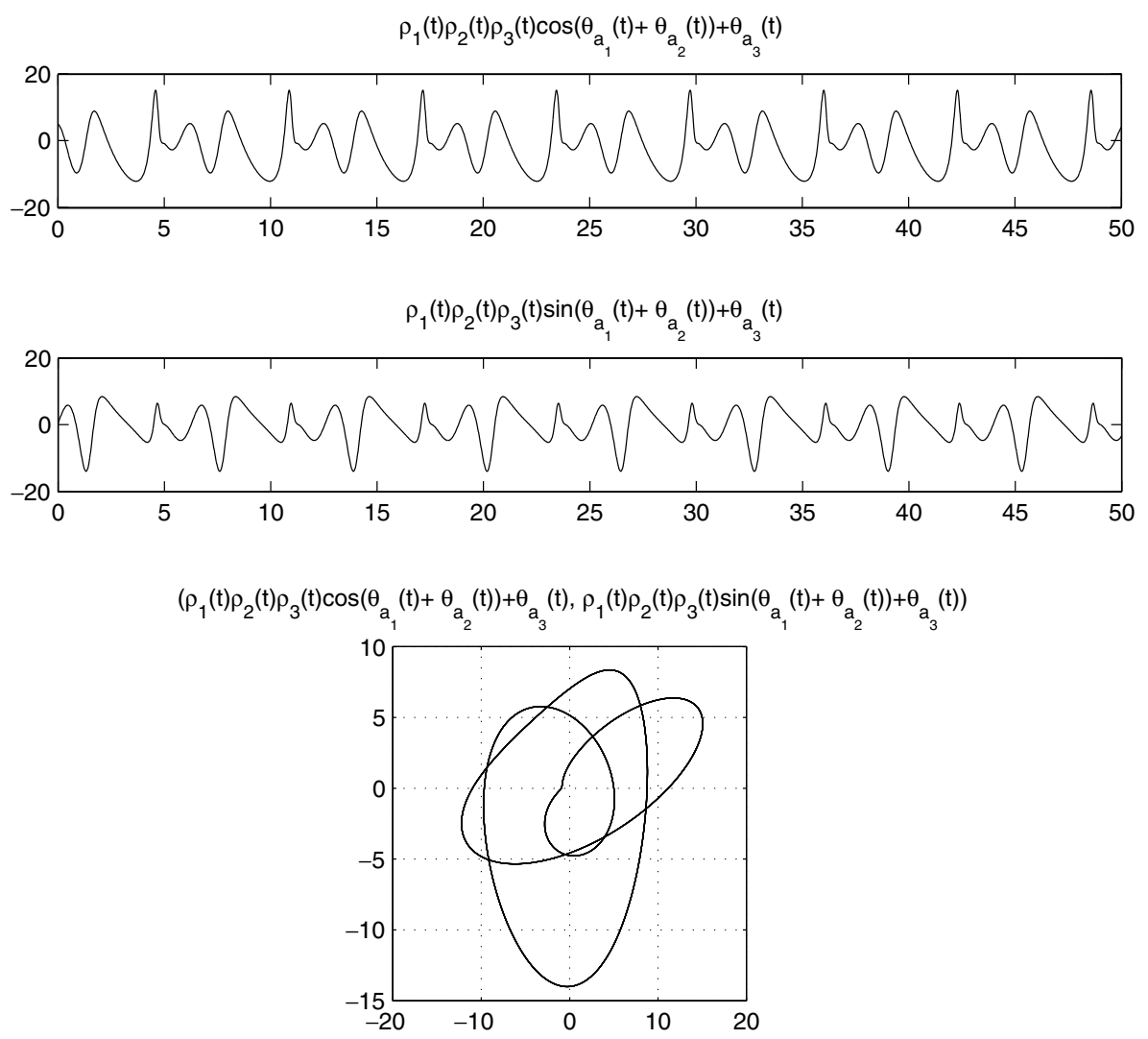

Fig. 5. Mono-component from product of three starlike functions.

where the part in the square bracket is $\rho_{1}$ and the outside part is $\cos \theta_{1}(t)$ and $\sin \theta_{1}(t)$, respectively, the constants $\rho_{0}, \theta_{0}, B$ are chosen so that $\rho_{1} \geq 0$.

Figures 6 and 7 are examples for $(3.6)$ for $\rho_{0}=1, r=1 / 2, \theta_{0}=3 \pi / 4, B=2$; and (3.8) for $\rho_{0}=1, r=1 / 2, \theta_{0}=\pi / 4, t_{a}=3 \pi / 2, B=2$, respectively.

\section{EMD vs Mono-Components}

It is a belief and practice in signal analysis that a signal is decomposed into the harmonic waves, and, in the complex analysis terms, into boundary values of power series and Laurent series. Mono-components, as generalization of trigonometric functions, are of a greater variety but without orthogonality. The observation based on recent development in practice of signal analysis ${ }^{6}$ and that in harmonic analysis ${ }^{5}$ suggest that adaptive decomposition of signals into monocomponents is possible. To further carry out the study it is a natural to ask how EMD goes with mono-components. More specifically, given a sum added up from a number of mono-components, at what extend EMD can reproduce the composing 
mono-components? A number of basic experiments are carried out to answer this question. In doing so, we stick on the believe that Fourier atoms and boundary values of starlike functions are among the fundamental constructive building blocks of signals.

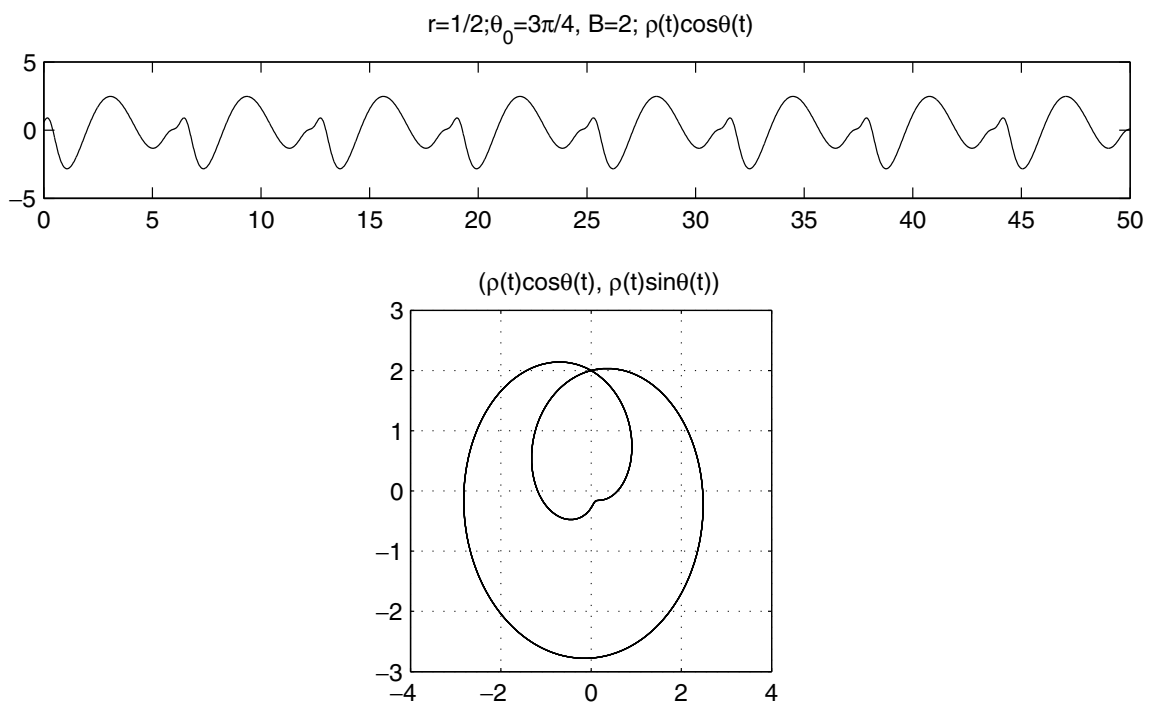

Fig. 6. Example for mono-components obtained from formula (3.6).
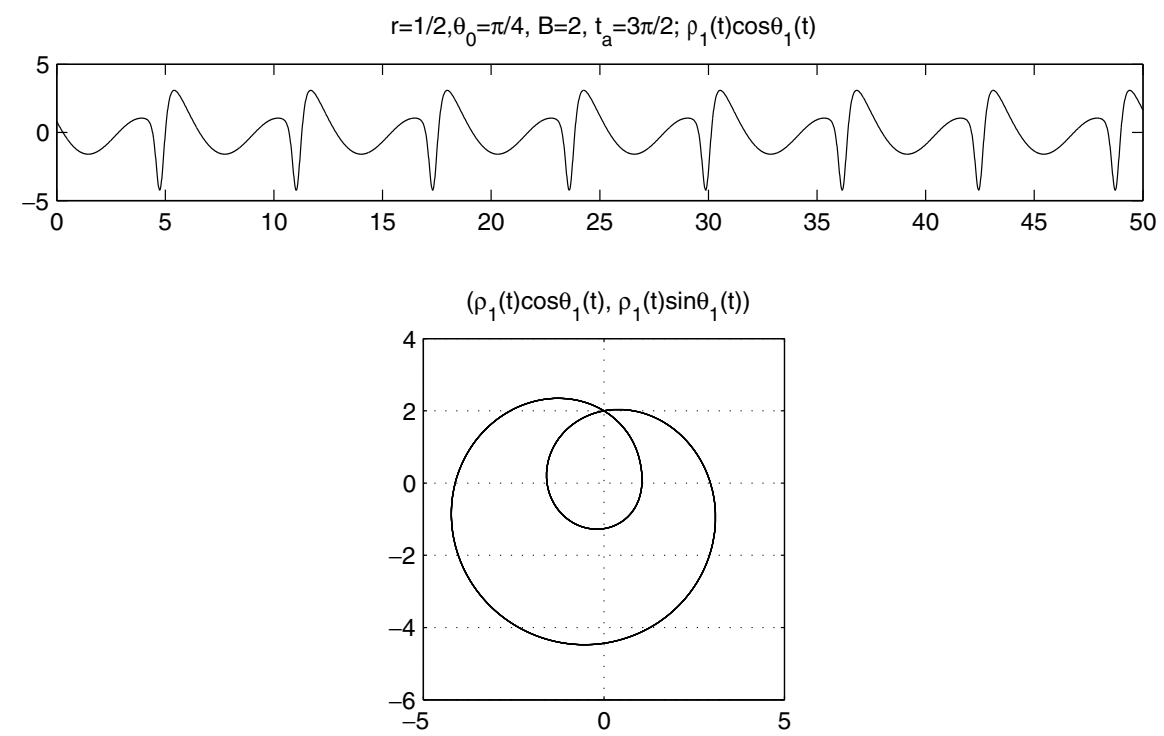

Fig. 7. Example for mono-components obtained from formula (3.8). 
Experiments and observations include:

(i) All Fourier atoms are IMFs. In fact, Fourier atoms cannot be further decomposed by the adopted EMD. A such experiment is presented in Fig. 8.

(ii) If the sum of a number of mono-components is itself an IMF, then it cannot be further decomposed by the EMD. This shows that EMD cannot decompose signals into their very fundamental constructive building blocks. The final results of EMD may still be combinations of those fundamental pieces. A such example is exhibited in Fig. 9.

(iii) EMD decomposes mono-components into its IMF pieces. In Fig. 10, we exhibit two examples. The first is the type of mono-components studied in Theorem (3.1): $f(t)=\left[7 / 4 \cos \theta_{1 / 2}(t)+2\right] \cos \theta_{1 / 2}(t)$; and the second: $f(t)=\rho(t) \cos \theta(t)$ in (3.6) is for $\rho_{0}=1, r=1 / 2, \theta_{0}=3 \pi / 4, B=2$. They exhibit that although mono-components are fundamental constructive building blocks, they are still decomposed into a number of IMFs pieces. What causes this is that those mono-components are not IMFs by themselves. Non-unimodular monocomponents are usually not IMFs.

(iv) In Fig. 11, we show that the sum of the two mono-components $2 \cos \left(\theta_{1 / 2+i / 2}(t)+\theta_{2 / 3}(t)\right)$ and $\cos \theta_{1 / 2}(t)$, both being IMFs, is decomposed by the EMD into a sum of another pair of non-trivial IMFs. $2 \cos \left(\theta_{1 / 2+i / 2}(t)+\right.$ $\left.\theta_{2 / 3}(t)\right)$ should be compared with the first IMF as they are the components of higher-frequency, while $\cos \theta_{1 / 2}(t)$ should be compared with the second IMF as they are the components of low-frequency. The two pairs look similar but not the same. This shows that EMD does not faithfully recover the composing fundamental pieces even though the latter being IMFs.

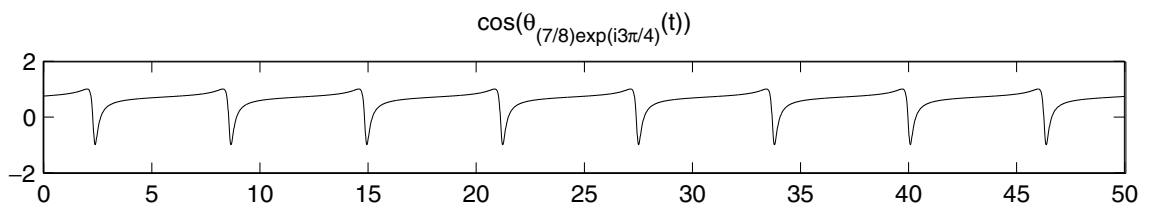

First Imf

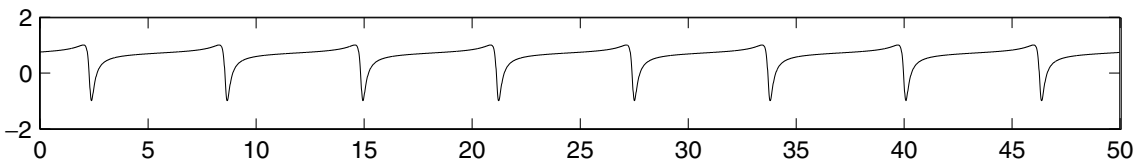

Residue

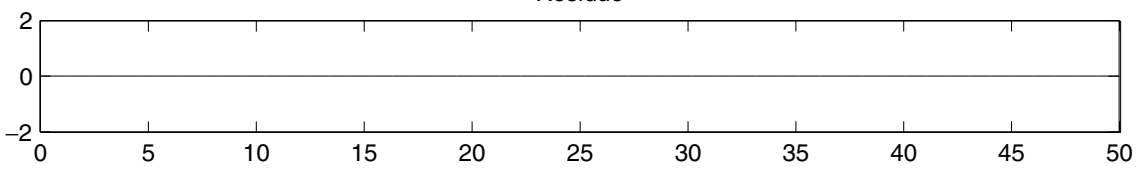

Fig. 8. Fourier atoms cannot be further decomposed by EMD. 

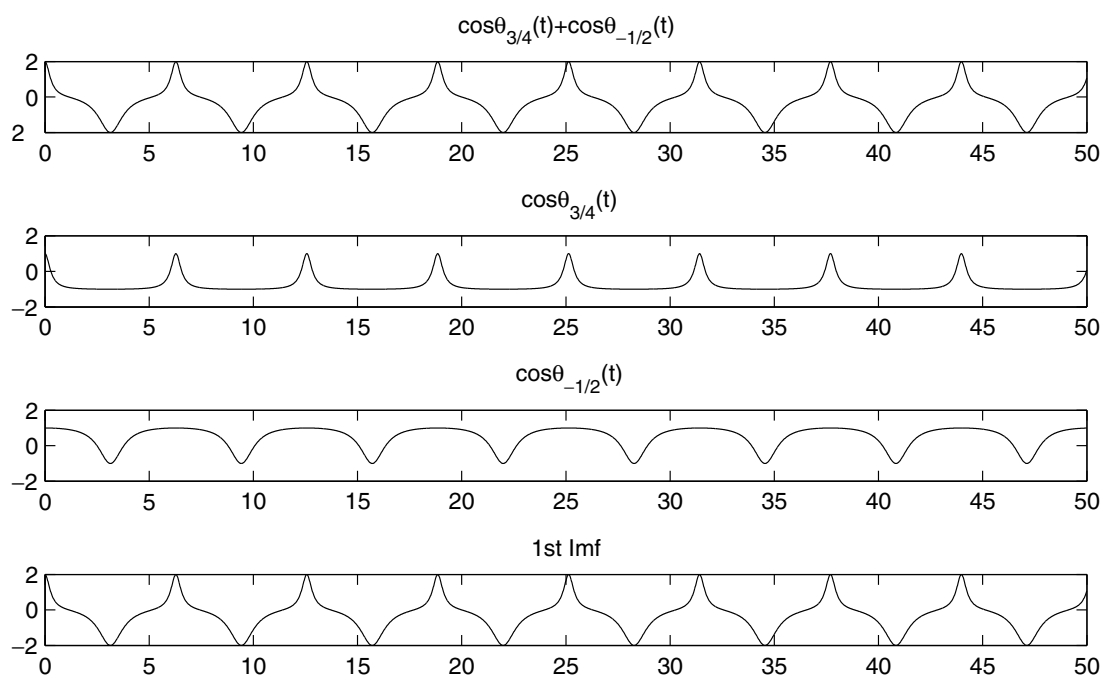

Residue

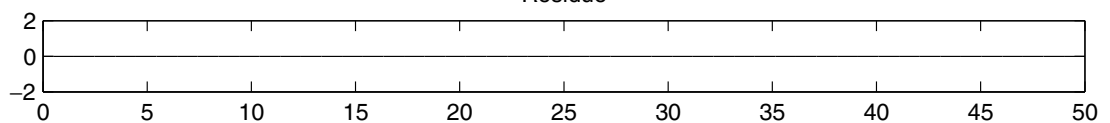

Fig. 9. If the sum is IMF, then it cannot be decomposed.
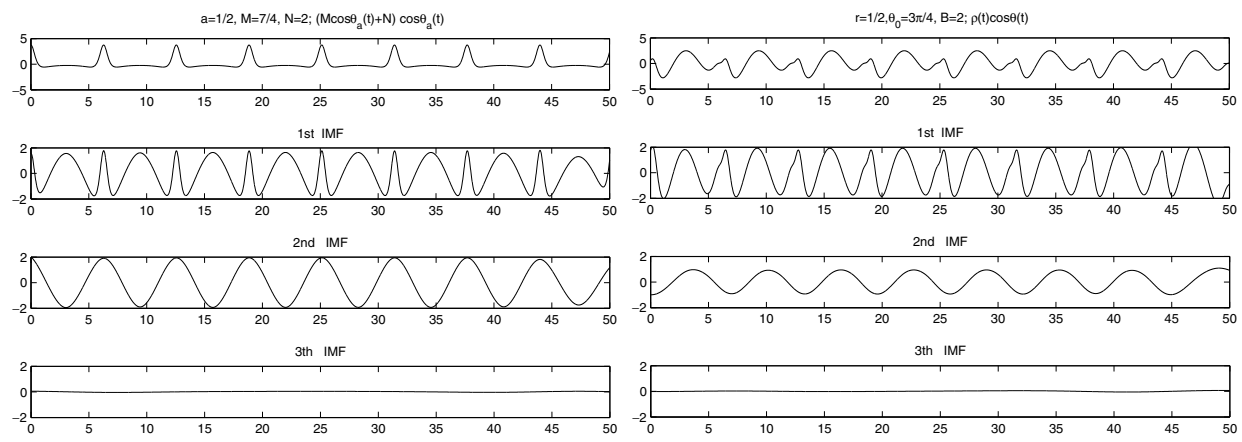

Fig. 10. EMD further decomposes mono-components.

(v) The situation exhibited in Fig. 12 is worse. In the experiment the sum of the two Fourier atoms $\cos \theta_{3 i / 4}(t)$ and $\cos \theta_{3 / 4}(t)$, both being IMFs, is decomposed into a pair of completely different IMFs. What happens there is that the two Fourier atoms attains the extreme values at different values of $t$ and thus their sum has more extreme values. The EMD gets the first IMF with more vibrations than any of the composing Fourier atoms and the second IMF does not look like any of the Fourier atoms either. 

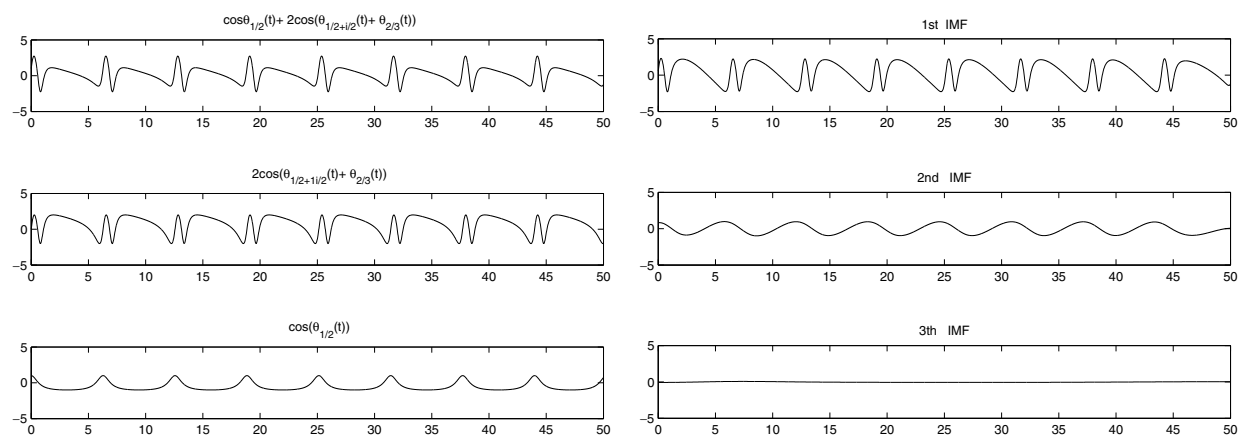

Fig. 11. EMD does not recover its own IMFs.
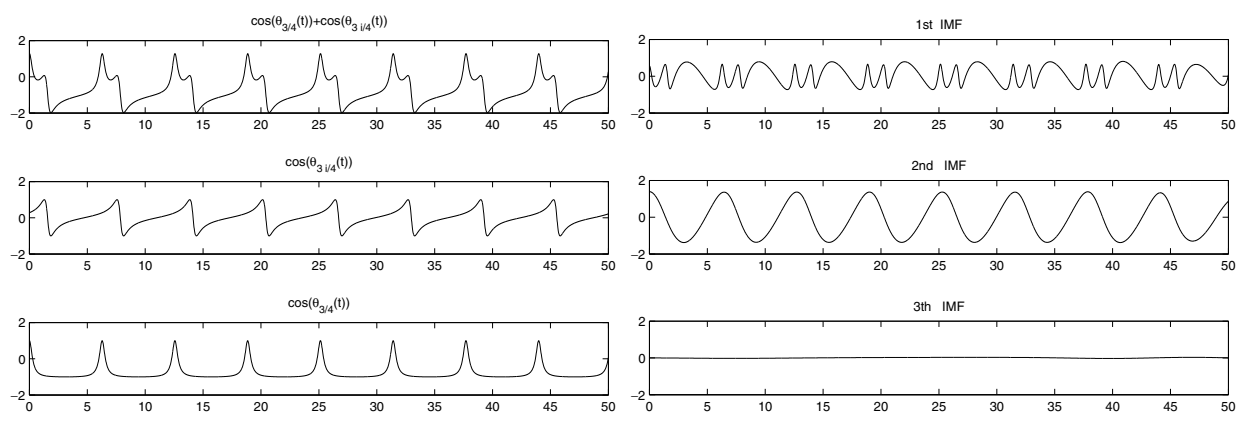

Fig. 12. Sum of two Fourier atoms that cannot be recovered by EDM.

\section{Conclusion}

Mono-components are defined to be the functions that possess analytic instantaneous frequencies. A fundamental class of mono-components consists of the boundary values of starlike functions, among which a more fundamental class consists of the Fourier atoms. The class of mono-components forms a non-orthogonal basis in $L^{2}$ that allows adaptive decomposition of the signals into sums of mono-components. The algorithm is to be sought.

IMFs are associated with the adopted EMD. They are outcomes of implementation of EMD. The algorithm EMD, on the other hand, depends on specially chosen functions in formulating envelops in the iteration procedure. In the sense of splineapproximation EMD is man-made, it is no wonder that IMFs fail to possess analytic instantaneous frequency, and EMD, therefore, is hard to faithfully decompose signals into the constructive pieces that possess analytic instantaneous frequencies.

\section{Acknowledgments}

Tao Qian and Liming Zhang are supported by Macao Science and Technology Development Fund 051/2005/A and research grant of the University of Macau No. 
RG079/04-05S/QT/FST. Hong Li is in part supported by the National Natural Science Foundations of China (NSFC) under grant 10771053. The authors would like to sincerely thank Haizhang Zhang, Rui Wang, Yuesheng Xu, Uwe Käuler, Paula Cerejelas, and Luoqing Li for some previous observations made together, and discussions.

\section{References}

1. E. Bedrosian, A product theorem for Hilbert transform, Proc. IEEE 51 (1963) 868-869.

2. L. Cohen, Time Frequency Analysis, Signal Processing Series (Prentice-Hall, 1995).

3. Q. H. Chen, L. Q. Li and T. Qian, Stability of frames generated by nonlinear Fourier atoms, Int. J. Wavelets Multiresolut. Inf. Process. 3 (2005) 465-476.

4. Q. H. Chen, L. Q. Li and T. Qian, Two Families of unit analytic signals with nonlinear phase, Phys. D 221 (2006) 1-12.

5. J. B. Garnett, Bounded Analytic Functions (Academic Press, 1987).

6. N. E. Huang et al., The empirical mode decomposition and the Hilbert spectrum for nonlinear and non-stationary time series analysis, Proc. R. Soc. London A 454 (1998) 903-995.

7. B. Picinbono, On instantaneous amplitude and phase of signals, IEEE Trans. Signal Process. 45(3) (1997) 552-560.

8. T. Qian, Analytic signals and harmonic measures, J. Math. Anal. Appl. 314 (2006) 526-536.

9. T. Qian, Characterization of boundary values of functions in Hardy spaces with application in signal analysis, J. Integral Equations Appl. 17 (2005) 159-198.

10. T. Qian, Mono-components for decomposition of signals, Math. Methods Appl. Sci. 29 (2006) 1187-1198.

11. T. Qian, Mono-component decomposition of signals, in Wavelet Analysis and Applications, eds. T. Qian, M. I. Vai and Y. Xu, Applied and Numerical Harmonic Analysis (Birkhäuser, 2006), pp. 315-335.

12. T. Qian, Q. H. Chen and L. Q. Li, Analytic unit quadrature signals with nonlinear phase, Phys. D 203 (2005) 80-87.

13. T. Qian, R. Wang, Y. S. Xu and H. Z. Zhang, Orthogonal bases with nonlinear phase, preprint.

14. T. Qian, Y. S. Xu, D. Y. Yan, L. X. Yan and B. Yu, Fourier spectrum characterization of Hardy spaces and applications, preprint.

15. R. C. Sharpley and V. Vatchev, Analysis of intrinsic mode functions, Constr. Approx. 24 (2006) $17-47$.

16. Y. S. Xu and D. Y. Yan, The Bedrosian identity for the Hilbert transform of product functions, Proc. Amer. Math. Soc. 134 (2006) 2719-2728.

17. B. Yu and H. Z. Zhang, The Bedrosian identity and homogeneous semi-convolution equations, to appear in J. Integral Equations Appl. 